\title{
Belphégor
}

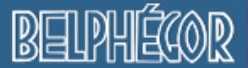

Littérature populaire et culture médiatique

18-2 | 2020

Roman Populaire Espagnol - Roman historique,

1900-1950

\section{Stratégies de marque et politiques éditoriales à destination des jeunes publics}

Hélène Laurichesse

\section{(2) OpenEdition}

Journals

Édition électronique

URL : http://journals.openedition.org/belphegor/3353

DOI : $10.4000 /$ belphegor.3353

ISSN : 1499-7185

Éditeur

LPCM

Référence électronique

Hélène Laurichesse, "Stratégies de marque et politiques éditoriales à destination des jeunes publics », Belphégor [En ligne], 18-2 | 2020, mis en ligne le 11 décembre 2020, consulté le 28 avril 2021. URL

http://journals.openedition.org/belphegor/3353 ; DOI : https://doi.org/10.4000/belphegor.3353

Ce document a été généré automatiquement le 28 avril 2021.

\section{(c)}

Belphégor est mis à disposition selon les termes de la Licence Creative Commons Attribution - Pas d'Utilisation Commerciale - Pas de Modification 4.0 International. 


\title{
Stratégies de marque et politiques éditoriales à destination des jeunes publics
}

\author{
Hélène Laurichesse
}

1 À l'ère de la convergence culturelle, la logique d'une offre transmédia constitue désormais un horizon de réflexion pour l'ensemble des acteurs des industries médiatiques et culturelles. Les artistes y voient un moyen d'expression créatif, les entreprises une opportunité économique (puisqu'elle génère des revenus supplémentaires) et stratégique (puisqu'elle permet de fidéliser les audiences), et les consommateurs l'occasion de varier leur expérience de réception et d'interactivité avec une œuvre. Les franchises médiatiques du type Harry Potter, Twilight ou Hunger Games répondent parfaitement en terme de contenus à ces logiques stratégiques. En déclinant leur récit sur plusieurs médias et en permettant le développement de licences d'exploitation pour les produits dérivés, elles allient les dimensions créative et marchande. Dans ce système assez largement dirigé vers la cible des adolescents et des jeunes adultes, le roman constitue, avec le film, l'un des éléments clefs du dispositif. Les exemples de franchises qui ont été cités trouvent leur origine dans la littérature jeunesse. Ce que nous souhaitons montrer à ce sujet, c'est que les stratégies éditoriales qui conduisent à cette conception transmédiatique de la consommation culturelle correspondent assez étroitement à celle de la marque : créer une préférence pour permettre l'identification (un repère), la différenciation (une identité), et la fidélisation (une relation dans le temps). Pourtant, s'il est désormais usuel de qualifier une entreprise médiatique de marque (une chaine de TV, une société de production), pour l'heure, il est encore rare d'envisager de la sorte une œuvre culturelle, fût-elle une franchise ${ }^{1}$. Pour approfondir cette visée stratégique, nous montrerons tout d'abord les similitudes entre la politique en œuvre dans les industries culturelles et la politique de marque en termes d'objectifs et de fonctions. Dans un deuxième temps, nous soulignerons le manque d'affichage de la marque en tant que telle dans ce contexte. D'une part, sa dénomination n'est pas assumée (on parle de franchise), d'autre part, elle n'est pas identifiée de la sorte. Cette perception biaisée de la marque renvoie à une 
méconnaissance généralisée de ce qu'est le concept de marque. Autrement dit, il est difficile aujourd'hui d'identifier ce qui est marque et ce qui ne n'est pas dans les dispositifs marketing contemporains. Le Brand Content et le marketing transmédia notamment, participent d'un mouvement général où les frontières entre l'artistique et le commercial s'estompent. Les contenus qui en résultent sont hybrides à l'image des stratégies qui les sous-tendent.

\section{Ceci est une marque}

Nous ne pouvons plus nous permettre de considérer la littérature comme un mode et une culture autonome, avec sa propre méthode de lecture, comme le montrent de récentes études sur les rapports entre les jeux et l'écriture. (Beavis, 2001; McClay, 2002; Mackereth et Anderson, 2000). Les livres évoluent dans un engouement cross-média, où l'intérêt des enfants s'étend à travers les romans, les films, les jeux vidéo, Internet et un ensemble de produits dérivés dignes de Star Wars. Nous devons alors penser comment s'agencent les différents types de lecture, comment ils se connectent, ce qu'ils ont en commun ${ }^{2}$.

2 Ce constat posé par Andrew Burn il y a 10 ans dépeint parfaitement, sous l'angle des préoccupations éditoriales, le contexte de culture de la convergence que Henry Jenkins introduisait sensiblement à la même époque ${ }^{3}$. Incarnée de manière emblématique par le transmedia storytelling qui propose une histoire à partir d'éléments dispersés sur différentes plateformes médiatiques, cette convergence est plus narrative que technologique. Elle traduit la nouvelle articulation qui s'opère entre le pôle des entreprises et celui des publics dans une forme de décloisonnement entre la production et la réception et entre les contenus de divertissements et ceux des marques. Elle renvoie à deux éléments fondamentaux: l'intertextualité (avec des textes qui se parlent) et la multimodalité (avec les différents supports mobilisés).

Cette nouvelle approche pose des questions de plusieurs ordres :

- l'éducation et l'enseignement à la lecture des médias (et plus seulement aux livres)

- les stratégies des éditeurs

- des attentes nouvelles des publics transmédiatiques qui se substituent aux publics monomédia (lecteurs, gamers, ou spectateurs).

4 Nous nous centrerons ici sur le deuxième problème en nous plaçant du point de vue des industries culturelles dans le contexte d'une offre surabondante, accentuée par les procédés numériques et la dématérialisation. Dans ce contexte, l'enjeu va être pour les producteurs de proposer des offres à la fois interactives, transmédias et ludiques et de gérer les relations avec les publics constitués en communautés.

On peut observer que le modèle du star-system, plus particulièrement associé à l'industrie cinématographique, prévaut dans l'ensemble des industries culturelles. Privilégiant une poignée d'artistes en termes de rémunération, carrière et notoriété, il s'est peu à peu élargi aux œuvres pour en favoriser quelques-unes au détriment de toutes les autres. Cette "stratégie du blockbuster ${ }^{4}$ » est selon Anita Elberse désormais adoptée par toutes les majors d'Hollywood, mais aussi par les éditeurs, les labels, et les producteurs de l'industrie de l'entertainement en général. Elle repose sur une forme de pari qui consiste à miser sur quelques titres phares au lieu de répartir les risques sur un catalogue diversifié. D’après les études réalisées par cette chercheuse, cette stratégie, fort risquée, n'en est pas moins payante d'un point de vue financier, à l'image de ce qu'est le «blockbuster » à l'origine (une arme de guerre ${ }^{5}$ ), écrasant tout sur son passage 
6 Ce processus est repris dans le modèle du Winner take all Society (WTA) de Frank et Cook ${ }^{6}$ avec pour point de départ une rémunération basée sur des performances relatives plutôt qu'absolues et une concentration de ces rémunérations sur quelques élus. La prédominance de ce modèle dans le domaine artistique, sportif, mais aussi universitaire peut s'expliquer par deux raisons principales.

7 La première est celle de l'incertitude sur le succès et la performance. On peut rappeler que dans le secteur culturel, le caractère unique de chaque œuvre ne permet pas de prévoir son succès. Alors que dans la plupart des secteurs industriels, les produits peuvent être évalués à partir de caractéristiques tangibles et objectives (leur confort, leur niveau de consommation d'énergie, leur taille, leur vitesse, leur performance, leur encombrement), dans le secteur culturel, les caractéristiques intangibles et subjectives entraînent des conditions de réussite incertaines. Cette imprévisibilité pèse sur le fonctionnement de l'ensemble des acteurs de la filière à partir de l'adage « personne ne sait $»^{7}$ : en l'occurrence, du point de vue de la production personne ne sait quels projets produire pour espérer avoir du succès ; de même, du point de vue de la consommation, personne ne sait quels contenus choisir parmi ceux proposés puisqu'ils sont à chaque fois uniques et originaux. D'où le repli des investisseurs et des publics sur des valeurs sûres, c'est à dire sur la notoriété et la célébrité pour faire face à ce doute.

La deuxième est celle de la non-substituabilité de ce type de biens, illustrée par la faible élasticité de la demande par rapport au prix. C'est ce qui explique que le spectacle onéreux d'une star pourra afficher complet pendant qu'un spectacle gratuit sera délaissé. Dans le domaine de l'édition, le contexte d'alignement des prix ne change pas la logique de «best-sellers» qui s'impose également avec un engouement fort des lecteurs occasionnels pour certains titres.

Or, comme l'a démontré Andréa Semprini ${ }^{8}$, ce modèle du WTA est aussi celui des marques : lorsque l'offre est abondante dans un contexte fortement concurrentiel, ce repère constitue un atout essentiel pour se différencier. Toutefois, envisager les contenus culturels à partir du modèle de la marque revient dans une certaine mesure à les considérer comme des produits, ou tout au moins des biens de consommation. Or, un tel rapprochement ne va pas de soi, ce qu'atteste une large littérature sur le sujet ${ }^{9}$. Leur singularité, qui se manifeste à la fois par la nature des biens, de leur production, et de leur consommation rend complexe le rapport des professionnels des industries culturelles à l'économie et au marketing.

Les biens culturels sont des biens uniques, « d'expérience » et les industries culturelles des industries "de prototypes », « de l'imaginaire ${ }^{10}$ ", qui allient reproductibilité et originalité des produits ${ }^{11}$. Leur production et leur consommation revoient à une "économie des singularités ${ }^{12}$ " qui se dessine à partir de ces caractéristiques et d'une forme d'autonomie de l'offre par rapport à la demande. Elle s'explique par la logique hédonique de la consommation culturelle correspondant à un besoin relatif, associé au divertissement, qui n'est pas utilitaire. Adapter la production à des besoins n'est pas réaliste dans ce secteur puisque le public ne sait pas ce qu'il recherche si ce n'est éprouver des émotions.

11 La logique d'une économie de l'offre complétée par celle d'un marketing de l'offre traduit l'idée que, dans ce secteur, l'offre ne dépend pas de la demande, mais crée la demande. Busson et Evrard en donnent la définition suivante : 
Le rôle des circuits de diffusion sera d'organiser la rencontre entre une œuvre et son public (marketing de l'offre) et non de définir une œuvre à partir des attentes supposées d'un public (marketing de la demande) ${ }^{13}$. « comme un risque calculé propre au métier (pour les professionnels) et à la sortie cinéma (pour les spectateurs) ${ }^{14}$ » conduit toutefois à "la nécessité d'envoyer des signaux aux individus quant à la qualité des objets en présence (prix labels, récompenses, etc..). Pour Nathalie Heinich $^{15}$, cette " économie de la visibilité » basée sur la loi du plus visible, représente une perspective de «devenir-marque » renvoyant à une vision de marchandisation de la culture. des dépenses marketing conséquentes à l'occasion de la sortie des livres, films, ou jeux vidéo, des références comme Harry Potter, Twilight ou Hunger Games visent à la fois l'objectif de visibilité et celui de repère. Ils sont un repère, car ils constituent un identifiant commercial qui permet de reconnaître les produits entre eux. Ces identifiants font l'objet d'un dépôt juridique qui permet de protéger des contrefaçons et de faire valoir la propriété. Dans un environnement médiatique saturé en informations, ces productions représentent également un imaginaire associé à ces signes distinctifs ( nom, logo, charte graphique, gingle, musique). Alors que pour les autres productions, il faut avoir lu le livre ou vu le film pour savoir quel est leur contenu, dans ces cas particuliers, le public sait à quoi s'attendre pour les différents épisodes à suivre. Les personnages sont familiers et l'univers aussi.

Ces productions sont aussi des marques, car elles permettent de générer des profits supplémentaires : le capital-marque représente la valeur dégagée par la marque par rapport à un produit équivalent (rendant le même service) sans marque, ou avec une marque à faible notoriété. Dans le secteur culturel, le licensing permet de dégager ces ressources avec la vente des produits dits dérivés. Les revenus du merchandising y sont d'ailleurs très largement supérieurs à ceux de toute autre exploitation. Ils auraient rapporté à Cars 8 milliards de dollars pendant que ceux de son exploitation cinématographique s'élevaient au total à environ 1 milliard de dollars ${ }^{16} ; 12$ milliards à James Bond soit, environ le double de la seule exploitation cinématographique ${ }^{17}$. La marque Star Wars représenterait au total 27 milliards de dollars, dont "seulement » 4 milliards ${ }^{18}$ issus de l'exploitation des sept films. Quant à Harry Potter, ses revenus atteindraient au total 24 milliards de dollars dont « seulement » 7,2 milliards de \$ pour les 8 films et 7,7 Milliards pour les ventes de livres ${ }^{19}$.

Dans cette dynamique, le rôle $\mathrm{du}$ film est essentiel puisque sa sortie a un effet multiplicateur au niveau des ventes. Si l'on prend le secteur de l'édition, le gain se situerait en moyenne entre 5 et 15 fois plus sur la vente des livres en comparant les ventes de la première semaine de sortie avec la moyenne des ventes des semaines précédentes ${ }^{20}$. Les livres du Seigneur des Anneaux auraient connu une augmentation de leur vente de $400 \%$ l'année qui a suivi la sortie du premier film en $2001^{21}$.

17 En raison de leurs spécificités, ces productions sont aussi des marques atypiques comme nous allons le voir. 


\section{Ceci n'est pas une marque} culturels présuppose un rapport complexe à la marque. En parallèle, l'économie de la visibilité également évoquée laisse penser que le secteur culturel est particulièrement prédisposé à une économie des marques. Cette ambivalence se traduit par un positionnement ambigu vis-à-vis de ces marques qui, en l'occurrence, ne sont pas dénommées comme telles. Il faut toutefois considérer cette analyse au regard de l'évolution paradigmatique de la discipline marketing. Elle permet de comprendre qu'il est devenu difficile d'identifier ce qui appartient aux logiques du marketing et ce qui n'en est pas. Même si la marque ne peut se résumer à sa seule dimension marketing, la compréhension de l'évolution de cette discipline est une clef permettant de comprendre les métamorphoses de la marque notamment sa tendance à la culturalisation. Sans perdre de vue l'objectif final (la transaction marchande) derrière les objectifs intermédiaires (la relation, l'expérience, la participation), il convient de prendre conscience que le marketing et la marque ne correspondent plus à la représentation caricaturale qu'en ont la grande majorité des non-spécialistes.

Le courant de la "Consumer Culture Theory» (CCT), labellisé par Arnould et Thompson en $2005^{22}$ synthétise vingt années de travaux sur le comportement de consommation, irrigués par des disciplines diverses (philosophie, sociologie, marketing, anthropologie, psychologie). Il apporte une vision alternative à l'approche rationnelle et positiviste du marketing pour étudier les choix de consommation. Il forge par ailleurs un nouvel ordre marketing illustré par trois pics paradigmiques ${ }^{23}$ qui ont eu une influence directe sur la stratégie de marque de ces deux dernières décennies: le marketing relationnel du début des années 90 , le marketing expérientiel de la fin des années 90 et le marketing collaboratif du milieu des années 2000.

Alors que la définition du marketing donnée par l'AMA (American Marketing Association) se centrait dans les années 1960 sur une vision instrumentale, "Le marketing est la mise en cuvre des actions destinées à diriger le flux des marchandises ou des services vers le consommateur", en 2013, on peut mesurer le chemin parcouru avec une vision désormais sociétale ${ }^{24}$ qui repose sur la cohabitation des dimensions sociales et managériales et l'impératif de création de valeur pour l'entreprise :

Le marketing est un processus social et managérial qui permet à des personnes ou à des organisations de créer de la valeur et de l'échanger avec d'autres, afin d'obtenir ce qu'elles recherchent et ce dont elles ont besoin. Dans le contexte plus étroit de l'entreprise, le marketing suppose l'établissement de relations d'échange rentables avec les clients, basées sur la notion de valeur.

21 Au début des années 90, le passage d'une approche transactionnelle à une approche relationnelle ${ }^{25}$ faite "d'expériences", de "réenchantement » et de co-création de valeur permet de comprendre le mouvement de culturalisation des marques. La marque détermine et est déterminée conjointement par ces transformations du marketing en se tournant vers le choix des moyens de communication les plus opportuns : si son principal canal d'expression était à l'origine la voie publicitaire, il s'accompagne désormais du Brand Content et de la Brand Culture. En découlent des contenus de marque et une culture de marque qui contribuent à rendre plus complexe l'identification de ce qui est marketing.

Belphégor, 18-2 | 2020 

rappelons-le, à insérer un produit dans un programme a priori non publicitaire (livre, film, jeu vidéo), en l'incorporant à la trame narrative contre rémunération ou en ce qui concerne le cinéma en avantages matériels (prêt de voiture, fourniture en bouteilles d'eau). 

polysémique de marque. Pour eux, il s'agit de "faire le d'argent", "vendre un produit ", "faire du business » ... Pour décrire les contenus évoqués (Harry Potter, Hunger Games, Twilight) ils n'ont tout simplement pas de mot adéquat. Le terme de franchise, utilisé par les professionnels (médias compris) leur est tout aussi étranger que celui de marque. Seule la terminologie de «saga » a été évoquée spontanément pour dénommer ces contenus. On notera d'ailleurs que le terme de franchise justement pose problème dans la mesure où les franchises médiatiques présentent des différences essentielles avec les franchises commerciales auxquelles est emprunté le concept. Le caractère « clandestin » de la marque médiatique se confirme donc à plus d'un titre. versus «ceci n'est pas une marque " sont, au-delà du clin d'œil, révélatrices d'une situation somme toute ambiguë. À l'évidence, la stratégie de marque s'affirme peu à peu dans certaines pratiques éditoriales, sans qu'elle ne soit explicitement assumée comme telle. On l'aura compris, le regard que nous proposons sur cette évolution éditoriale n'est ni alarmiste ni militant. Il ne s'agit pas de défendre une idéologie ni un genre de production en particulier, mais simplement de mettre en lumière un 
phénomène à l'oeuvre. Les productions que nous évoquons notamment, s'affichent comme une forme de genre en elles-mêmes, en coexistant au côté d'autres quantitativement bien plus nombreuses. Les résultats de l'étude menée par Charles R. Acland $^{33}$ qui a travaillé en collaboration avec l'Institut Statistique à l'Unesco (ISU) pour appréhender l'industrie cinématographique peuvent très certainement s'étendre à l'ensemble des industries culturelles. Ils montrent que si économiquement les blockbusters prédominent le marché, leurs productions, d'un point de vue quantitatif, ne sont pas pour autant représentatives de l'ensemble des œuvres. En conclusion, le chercheur souligne "La culture cinématographique n'est pas en voie de disparition, mais plutôt en train d'émerger sous la forme de différentes strates». Ces nouvelles formes répondent tout simplement à une évolution des pratiques de consommation directement liées aux nouvelles technologies de l'information et de la communication. On peut aussi admettre que la vision transmédiatique qu'elles introduisent peut être par ailleurs ambitieuse et créative. Ici comme ailleurs, il convient de ne pas faire de généralisation, le talent et la qualité peuvent se trouver dans toutes les formes d'expression artistique, tout comme la médiocrité n'est pas non plus caractérisée par un genre en particulier. Cette logique s'inscrit bien dans les exigences de l'arbitrage entre innovation et répétition au sens d'Umberto $\mathrm{Eco}^{34}$, ou encore de la dialectique " standardisation-différenciation ${ }^{35}$ » de Laurent Creton. L'innovation et la nécessité d'un renouvellement permanent restent les principales sources de différenciation, avec l'idée que « le succès résulte le plus souvent de la transgression des cadres de référence, tout en s'appuyant sur des logiques de continuité: un art de la combinaison des normes et des logiques de novation ${ }^{36} »$.

\section{NOTES}

1. Laurichesse H., La stratégie de marque dans l'audiovisuel, Paris, Armand Colin Recherches, 2013.

2. Burn A. 2005. « Potter-Literacy - from book to game and back again ; literature, film, game and cross-media literacy » in Papers : Explorations into Children's Literature, vol.14, n³, p 5-17. Republié dans Contemporary Literary Criticism, vol. 217, April 2006

3. Jenkins, H., Convergence Culture: Where Old and New Media Collide. New York, New York University Press, 2006.

4. Elberse A. Blockbusters, Why Big Hits - and Big Risks - are the Future of Entertainment Business, Londres, Faber \& Faber Non-Fiction, 2014, p. 8.

5. Le terme désigne à l'origine une bombe utilisée pendant la Seconde Guerre mondiale par l'aviation britannique pour détruire des pâtés de maison (block).

6. L'expression peut être traduite par «Le Gagnant rafle tout ». Frank R., Cook P., The WinnerTake-All-Society, New York, Free Press, 1995.

7. Caves R.E., Creative Industries, Contracts between arts and commerce, Harvard, Harvard University Press, 2002.

8. Semprini A., La marque, une puissance fragile, Paris, Vuibert, 2005.

9. Benhamou F. L'économie de la culture, 7ème édition, Paris, Éditions la découverte, 2011, Bourgeon-Renault D., Gombault A., Petr C., Debenedetti S., Marketing de l'Art et de la Culture : Spectacle vivant, patrimoine et industries culturelles, Paris, Dunod, 2009, Evrard Y., Le management des 
activités artistiques et culturelles, Paris, Economica, 1993, Evard Y., Bourgeon D., Petr C., «Le comportement de consommation culturelle : un état de l'art ", Actes du 16ème Congrès International de l'AFM, Tome 1, p.183-202, 2000, Frey B. S., Arts and Economics: Analysis and Cultural Policy, Springer, New York, Greffe, X. 2002. Arts et artistes au miroir de l'économie, Paris, Economica, 2000.

10. Flichy P., Les industries de l'imaginaire, Grenoble, PUG, 1991.

11. Benhamou F. L'économie de la culture, 7ème édition, Paris, Éditions La Découverte, 2004. p. 66.67 .

12. Karpik L. L'économie des singularités, Paris, Gallimard, 2007.

13. Busson A., Evrard Y., Les industries culturelles et créatives, Économie et stratégie, Paris, Vuibert, 2013. p.9

14. Gimello Mesplomb F., "Trois nouvelles «zones grises» au prisme des évolutions sociotechniques du cinéma : économie de la notoriété, incertitude et phénomènes de traînes ", Les cahiers de l'affecav, 2012 [En ligne] http://map.revues.org/762.

15. Heinich N., De la visibilité, Excellence et singularité en régime médiatique, Paris, Gallimard, 2012. p. 360

16. Le montant exact s'élève à 1021823 35. Georg Szalai, « Disney: 'Cars' has crossed $\$ 8$ billion in global retail sales ", The Hollywood Reporter, 14 février 2011

17. Devilliers S. James Bond, La machine de guerre publicitaire. Site France Inter, émission Le grand bain. [En ligne] http://www.franceinter.fr/emission-le-grand-bain-james-bond-la-machine-deguerre-publicitaire. Samedi 20 Octobre 2012 [consulté le 27/09/2020]

18. Le montant exact s'élève à 4277000000 . http://www.statisticbrain.com/star-wars-totalfranchise-revenue/

19. [En ligne] http://www.statisticbrain.com.

20. «Cinéma : La licence sort le grand jeu en magasin ». Kazachock Mag, Octobre-Décembre 2010.

21. Benhamou F. Ibid., p.199.

22. Arnould, E.J., Thompson, C.J. « Consumer Culture Theory (CCT): Twenty Years of Research ». Journal of Consumer Research, Vol.31, $\mathrm{n}^{\circ} 4,2005$, p. 868-882.

23. Cova B., Louyot-Gallicher M., Bonnemaison A. Marketing critique : le consommateur collaborateur en question, Paris, Éditions Lavoisier, « ERDF R \&D », 2010.

24. Kotler P., Armonstrong G., Le Nagard E., Lardinoit T. Principes de marketing, 11ème édition, Paris, Pearson, 2013.

25. Hezel P. 1996. Le marketing relationnel, Paris, Presse Universitaires de France, «Que sais-je ?», p. 18

26. Bô D. Du Brand Content à la Brand Culture. Les Clefs d'une stratégie de contenu, Vol 2. , Paris, QualiQuanti, 2012. p.3. [en ligne] http://www.leslivresblancs.fr.

27. On citera la saga Lady Dior réalisée sous forme de 4 courts métrages avec Marion Cotillard filmé par David Lynch chacun portant le nom d'une ville et accompagnant le lancement d'une nouvelle couleur du Lady Dior. Le magazine digital crée par Activia intitulé " Mon ventre le Mag » pour trouver des conseils nutritionnels ; l'application gratuite «J'M la cuisine » proposée par Ma Vie en Couleurs (groupe Mondelez) avec de nombreuses recettes.

28. Holt D.B. 2004. How Brands Become Icons, Harvard Business Review Press ; Holt D., Cameron D. Cultural Strategy, Oxford, OUP Oxford, 2012 ; Schroeder J.E., Salzer-Morling M. 2006. Brand Culture, Routledge in Bô D. in Brand Culture - Développer le potentiel des marques, Paris, Editions Dunod, 2013. 29. Elliot R., Davies A. « Symbolic Brand and Authenticity of Identity Performance », in Schroeder J.E., Salzer-Morling M.(dir.) Brand Culture, p.138-153, Londres, Routledge, 2006.

30. Articles L. 121-1I et L. 121 1II du code de la consommation.

31. https://www.youtube.com/watch?v=aM70zDrERfw

32. Laurichesse, H. Quand les films deviennent des marques, Au croisement de la "culturalisation " des marques et du branding des industries culturelles, Habilitation à Diriger les Recherches, Université Toulouse Jean-Jaurès, 2 Avril 2015. 
33. Acland C. R. "Des superproductions internationales aux succès nationaux ", Bulletin d'information de l'ISU n, Février 2012.

34. Eco U., «Innovation répétition : entre esthétique moderne et post-moderne ». Réseaux, 1994, Vol. $12 \mathrm{n}^{\circ} 68$, p.9-26.

35. Creton L., Economie du cinéma: Perspectives stratégiques, 5ème édition, Paris, Armand Colin, 2014. p.47.

36. Creton. Ibid. p.47. 